\title{
Erratum
}

Kevin M. Johnson*

\section{Erratum to: Using Bayes' rule in diagnostic testing: a graphical explanation}

https://doi.org/10.1515/dx-2017-9011

Erratum to: Kevin M. Johnson. 2017. Using Bayes' rule in diagnostic testing: a graphical explanation. Diagnosis. Volume 4, Issue 3, pages 159-167. (DOI 10.1515/ dx-2017-0011):

On page 162 , line 5 , the sentence was incorrect. The correct sentence should be "Specificity is defined as the proportion (or percentage) of patients without the disorder who have a negative test result".

On page 163 , line 17 , the sentence was incorrect. The correct sentence should be "Negative predictive value is defined as the proportion (or percentage) of patients with a negative test result who do not have the disorder". 